\title{
Controlling chaotic systems via time-delayed control
}

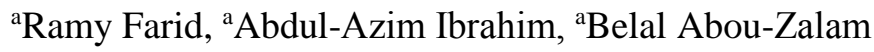 \\ ${ }^{a}$ Faculty of electronic engineering, department of industrial electronics and control Menofia \\ University, Menuf, Egypt
}

\begin{abstract}
Based on Lyapunov stabilization theory, this paper proposes a proportional plus integral time-delayed controller to stabilize unstable equilibrium points (UPOs) embedded in chaotic attractors. The criterion is successfully applied to the classic Chua's circuit. Theoretical analysis and numerical simulation show the effectiveness of this controller.

Index Terms - Chaotic systems, proportional plus integral time-delayed controller, Taylor approximation.
\end{abstract}

\section{INTRODUCTION}

$\mathrm{D}$ YNAMIC chaos is a very interesting non-linear effect which has been intensively studied in science and engineering. The effect is very common, it has been detected in a large number of dynamic systems of various physical nature. However, this effect is usually irregular, complex and undesirable in practice, and it restricts the operating range of many electronic and mechanic devices. Recently, controlling this kind of complex dynamical systems has attracted a great deal of attention within the engineering society. Chaos control, in a broader sense, can be divided into two categories: one is to suppress the chaotic dynamical behavior [1-12] and the other is to generate or enhance chaos in nonlinear systems $[13,14]$. Nowadays, different techniques and methods have been proposed to achieve chaos control. Among many methods, the time delayed feedback control DFC method [1]. This method utilizes the difference between the states and the delayed states as an input control provided that the delayed time is determined as the period of the unstable periodic orbits UPO to be stabilized. Furthermore $[2,3]$ also proposed a DFC based controller to stabilize the

Manuscript received October 1, 2007. (Write the date on which you submitted your paper for review.) This work was supported in part by the U.S. Department of Commerce under Grant BS123456 (sponsor and financial support acknowledgment goes here). Paper titles should be written in uppercase and lowercase letters, not all uppercase. Avoid writing long formulas with subscripts in the title; short formulas that identify the elements are fine (e.g., "Nd-Fe-B"). Do not write "(Invited)" in the title. Full names of authors are preferred in the author field, but are not required. Put a space between authors' initials.

F. A. Author is with the National Institute of Standards and Technology, Boulder, CO 80305 USA (corresponding author to provide phone: 303-555-5555; fax: 303-555-5555; e-mail: author@ boulder.nist.gov).

S. B. Author, Jr., was with Rice University, Houston, TX 77005 USA. He is now with the Department of Physics, Colorado State University, Fort Collins, CO 80523 USA (e-mail: author@lamar. colostate.edu).

T. C. Author is with the Electrical Engineering Department, University of Colorado, Boulder, CO 80309 USA, on leave from the National Research Institute for Metals, Tsukuba, Japan (e-mail: author@nrim.go.jp).
UPOs by virtue of the iterative learning control strategy. A time-delayed integrity controller is proposed in [4] to ensure the stabilization of UPOs in the case of sensor failures. Sliding mode control of uncertain unified chaotic systems is proposed in [5] based on a proportional plus integral sliding surface. dislocated and enhancing feedback control [6,7] which multiply the independent variable of the system function with coefficient and take the result as feedback gain (the same coefficient for all states) based on Jacobi matrix, speed feedback control [6-8] multiply the derivative of independent variable with coefficient. And other feedback control techniques [9-12]. At the same time, chaos synchronization also is an important topic, and has obtained a lot of availability results [15-19].

The aim of this paper is to proposed new scheme of time-delayed controller based on proportional plus integral (PI) to stabilize unstable equilibrium points (UPOs) embedded in chaotic attractors based on Lyapunov stabilization theory.

The reset of the letter is organized as follows. In Section 2 the control problem is stated. In Section 3 PI time-delayed controller is proposed to stabilize UPOs using Lyapunov stabilization theory. The proposed controller is applied with numerical simulation to the classic Chua's circuit in section 4 . Finally, some conclusions are given in section 5.

\section{Problem Statement}

Considering a chaotic system with state equation in the form

$$
\dot{x}=A x+g(x)
$$

Where $x \in R^{n}$ is the state vector, $A \in R^{n x n}$ is constant matrix and $g(x)$ is a nonlinear vector on the state vector $x$. Assuming that

$$
g(x)-g(\tilde{x})=M_{x, \tilde{x}}(x-\tilde{x})
$$

For a bounded matrix $M_{x, \tilde{x}}$ in which the elements are dependent on $x$ and $\tilde{x}$. Most of the chaotic systems can be described by (1) and (2).

General speaking, chaotic systems can be decomposed into a linear part and nonlinear function vector part.

Among many chaotic systems,

Lorenz system [14] 
$\left[\begin{array}{c}\dot{x} \\ \dot{y} \\ \dot{z}\end{array}\right]=\left[\begin{array}{ccc}-\sigma & \sigma & 0 \\ r & -1 & 0 \\ 0 & 0 & -\rho\end{array}\right]\left[\begin{array}{l}x \\ y \\ z\end{array}\right]+\left[\begin{array}{c}0 \\ -x z \\ x y\end{array}\right]$

RÖssler system [14]

$$
\left[\begin{array}{c}
\dot{x} \\
\dot{y} \\
\dot{z}
\end{array}\right]=\left[\begin{array}{ccc}
0 & -1 & -1 \\
1 & a & 0 \\
0 & 0 & -b
\end{array}\right]\left[\begin{array}{l}
x \\
y \\
z
\end{array}\right]+\left[\begin{array}{c}
0 \\
0 \\
c+x z
\end{array}\right]
$$

Chua system [14]

$$
\left[\begin{array}{c}
\dot{x} \\
\dot{y} \\
\dot{z}
\end{array}\right]=\left[\begin{array}{ccc}
-\alpha & \alpha & 0 \\
1 & -1 & 1 \\
0 & -\beta & 0
\end{array}\right]\left[\begin{array}{l}
x \\
y \\
z
\end{array}\right]+\left[\begin{array}{c}
\alpha f(x) \\
0 \\
0
\end{array}\right]
$$

With

$$
f(x)=b x+\frac{1}{2}(a-b)(|x+1|-|x-1|)
$$

have the same form as Eq. (1).

Our problem undertaken here is to construct a controller

$$
u(t)=\left(u_{1}(t), u_{2}(t), \ldots . ., u_{n}(t)\right)^{T} \in R^{n \times 1}
$$

to stabilize the UPOs within chaotic attractors. Therefore the controlled chaotic system can be described by

$$
\dot{x}(t)=A x(t)+g(x(t))+u(t)
$$

Suppose that the UPO to be stabilized is T-periodic, i.e.

$$
\dot{x}(t-T)=A x(t-T)+g(x(t-T))
$$

Defining the state error as

$$
e(t)=x(t)-x(t-T)
$$

The error dynamics is

$$
\dot{e}(t)=A e(t)+g(x(t))-g(x(t-T))+u(t)
$$

Based on Eq. (2)

$$
\dot{e}(t)=\left(A+M_{x(t), x(t-T)}\right) e(t)+u(t)
$$

With the help of the controller $u(t)$, the problem of stabilization of the T-periodic orbit becomes the problem of stabilization of Eq. (9) to either a periodic or equilibrium points.

\section{CONTROLLER DESIGN}

A proportional plus integral time-delayed controller is proposed to stabilize UPOs embedded in chaotic attractors. Controller $u(t)$ is chosen as

$$
u(t)=-k_{p}(x(t)-x(t-T))-k_{i} \int_{t_{1}}^{t_{2}}(x(t)-x(t-T)) d t
$$

Where $k_{p}$ and $k_{i}$ are diagonal matrices with diagonal gain elements $k_{p 1}, k_{p 2}, \ldots . ., k_{p n} \quad$ and $\quad k_{i 1}, k_{i 2}, \ldots . ., k_{i n}$ respectively.

Theorem 1 if controller $u(t)$ is constructed as Eq. (10), then the error system (9) is globally exponentially stable for $T<<1$.if there exists a positive definite symmetric constant matrix $P$ such that

$$
\begin{aligned}
& \left(A+M_{x(t), x(t-T)}-k_{p}-T k_{i}\right)^{T} P \\
& +P\left(A+M_{x(t), x(t-T)}-k_{p}-T k_{i}\right)
\end{aligned}
$$

Where $\mu$ denotes a negative constant, and $I$ is the identity matrix

\section{Proof}

For $T<<1, \quad x(t-T)=x(t)-\dot{x}(t) T+o\left(t^{2}\right)$, then by Taylor approximation, we have $x(t)-x(t-T)=\dot{x}(t) T$, so the controller $u(t)$ of Eq. (10) becomes

$$
u(t)=-k_{p}(x(t)-x(t-T))-k_{i} T \int_{t 1}^{t 2} \dot{x}(t) d t
$$

For $t_{2}=t$ and $t_{1}=t-T$

$u(t)=-k_{p}(x(t)-x(t-T))-k_{i} T((x(t)-x(t-T)))$

$u(t)=-k_{p} e(t)-k_{i} T e(t)$

By constructing (13) into (9)

$$
\dot{e}(t)=\left(A+M_{x(t), x(t-T)}-k_{p}-T k_{i}\right) e(t)
$$

Now choose the Lyapunov function

$$
V=e^{T} P e
$$

where $P$ is a positive definite symmetric constant matrix.

Then its derivative is

$\dot{V}=\dot{e}^{T} P e+e^{T} P \dot{e}$

$=\left[\left(A+M_{x(t), x(t-T)}-k_{p}-T k_{i}\right) e\right]^{T} P e$

$+e^{T} P\left[\left(A+M_{x(t), x(t-T)}-k_{p}-T k_{i}\right) e\right]$

$=e^{T}\left[\left(A+M_{x(t), x(t-T)}-k_{p}-T k_{i}\right)^{T} P\right.$

$\left.+P\left(A+M_{x(t), x(t-T)}-k_{p}-T k_{i}\right)\right] e$

$\leq \mu\|e\|^{2}<0$

where $\|\cdot\|$ denotes the Euclidean norm.

\section{NUMERICAL SIMULATION}

To demonstrate the use of chaos control criterion proposed herein, Chua's circuit is considered as an example of chaotic systems.

\section{A. Chua's circuit}

Chua's circuit can be described by (5) and (6), where $\alpha>0$, $\beta>0, a<b<0, f($.$) is a piecewise linear function.$ Chua's circuit exhibits a chaotic behavior 
for $\alpha=9.78, \beta=14.97, a=-1.31$ and $b=-0.75$ as shown in Fig. 1, and in Eq. (6), we have

$$
f(x(t))-f(x(t-T))=k_{x(t), x(t-T)}(x(t)-x(t-T))
$$

Where $k_{x(t), x(t-T)}$ is dependent on $x(t)$ and $x(t-T)$, and varies in the interval $[a, b]$ for $t \geq 0$ that is, $k_{x(t), x(t-T)}$ is bounded by the condition of $a \leq k_{x(t), x(t-T)} \leq b<0$ graphical representation of $f(x)$ in [14].
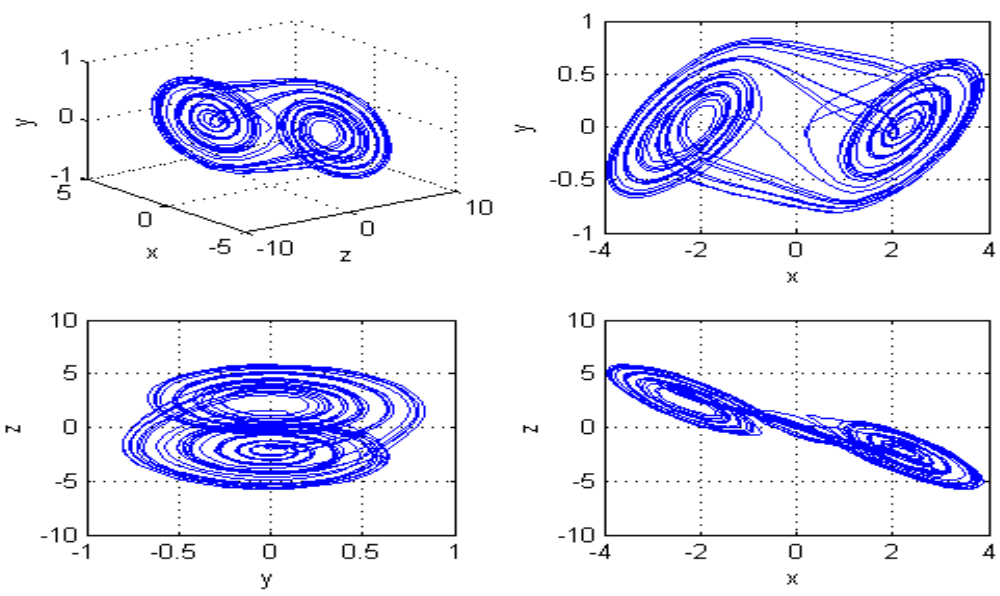

Fig. 1. The attractors of Chua's circuit

System (5) has the same form of Eq. (1) with

$$
A=\left[\begin{array}{ccc}
-\alpha & \alpha & 0 \\
1 & -1 & 1 \\
0 & -\beta & 0
\end{array}\right], g(x(t))=\left[\begin{array}{c}
-\alpha f(x(t)) \\
0 \\
0
\end{array}\right]
$$

Consider

$$
\begin{aligned}
& g(x(t))-g(x(t-T))=\left[\begin{array}{c}
-\alpha(f(x(t)-f(x(t-T)) \\
0 \\
0
\end{array}\right]=\left[\begin{array}{c}
-\alpha k_{x(t), x(t-T)}(x(t)-x(t-T)) \\
0 \\
0
\end{array}\right] \\
& =\left[\begin{array}{ccc}
-\alpha k_{x(t), x(t-T)} & 0 & 0 \\
0 & 0 & 0 \\
0 & 0 & 0
\end{array}\right]\left[\begin{array}{c}
x(t)-x(t-T) \\
y(t)-y(t-T) \\
z(t)-z(t-T)
\end{array}\right] \\
& =M_{x(t), x(t-T)} e(t)
\end{aligned}
$$

Where

$$
M_{x(t), x(t-T)}=\left[\begin{array}{ccc}
-\alpha k_{x(t), x(t-T)} & 0 & 0 \\
0 & 0 & 0 \\
0 & 0 & 0
\end{array}\right]
$$

From Eqs. (14) and (18), we get 


$$
A+M_{x(t), x(t-T)}-k_{p}-T k_{i}=\left[\begin{array}{ccc}
-\alpha-k_{p 1}-T k_{i 1}-\alpha k_{x(t), x(t-T)} & \alpha & 0 \\
1 & -1-k_{p 2}-T k_{i 2} & 1 \\
0 & -\beta & -k_{p 3}-T k_{i 3}
\end{array}\right]
$$

Choosing

$$
P=\left[\begin{array}{ccc}
p_{1} & 0 & 0 \\
0 & p_{2} & 0 \\
0 & 0 & p_{3}
\end{array}\right]
$$

where $p_{1}, p_{2}$ and $p_{3}$ are positive constants, then

$$
\begin{aligned}
& \left(A+M_{x(t), x(t-T)}-k_{p}-T k_{i}\right)^{T} P+P\left(A+M_{x(t), x(t-T)}-k_{p}-T k_{i}\right)-\mu I \\
& =\left[\begin{array}{ccc}
-2 p_{1}\left(\alpha+k_{p 1}+T k_{i 1}+\alpha k_{x(t), x(t-T)}+\frac{\mu}{2 p_{1}}\right) & p_{1} \alpha+p_{2} & 0 \\
p_{1} \alpha+p_{2} & -2 p_{2}\left(1+k_{p 2}+T k_{i 2}+\frac{\mu}{2 p_{2}}\right) & p_{2}-p_{3} \beta \\
0 & p_{2}-p_{3} \beta & -2 p_{3}\left(k_{p 3}+T k_{i 3}+\frac{\mu}{2 p_{3}}\right)
\end{array}\right]
\end{aligned}
$$

In Eq. (21), we have

$$
\begin{aligned}
& \Delta_{1}=-2 p_{1}\left(\alpha+k_{p 1}+T k_{i 1}+\alpha k_{x(t), x(t-T)}+\frac{\mu}{2 p_{1}}\right) \\
& (22) \Delta_{2}=\operatorname{det}\left[\begin{array}{cc}
-2 p_{1}\left(\alpha+k_{p 1}+T k_{i 1}+\alpha k_{x(t), x(t-T)}+\frac{\mu}{2 p_{1}}\right) & p_{1} \alpha+p_{2} \\
p_{1} \alpha+p_{2} & -2 p_{2}\left(1+k_{p 2}+T k_{i 2}+\frac{\mu}{2 p_{2}}\right)
\end{array}\right] \\
& =4 p_{1} p_{2}\left(\alpha+k_{p 1}+T k_{i 1}+\alpha k_{x(t), x(t-T)}+\frac{\mu}{2 p_{1}}\right)\left(1+k_{p 2}+T k_{i 2}+\frac{\mu}{2 p_{2}}\right)-\left(p_{1} \alpha+p_{2}\right)^{2} \\
& \Delta_{3}=\operatorname{det}\left[\begin{array}{ccc}
-2 p_{1}\left(\alpha+k_{p 1}+T k_{i 1}+\alpha k_{x(t), x(t-T)}+\frac{\mu}{2 p_{1}}\right) & p_{1} \alpha+p_{2} & 0 \\
p_{1} \alpha+p_{2} & -2 p_{2}\left(1+k_{p 2}+T k_{i 2}+\frac{\mu}{2 p_{2}}\right) & p_{2}-p_{3} \beta \\
0 & p_{2}-p_{3} \beta & -2 p_{3}\left(k_{p 3}+T k_{i 3}+\frac{\mu}{2 p_{3}}\right)
\end{array}\right] \\
& =2 p_{1}\left(p_{2}-p_{3} \beta\right)^{2}\left(\alpha+k_{p 1}+T k_{i 1}+\alpha k_{x(t), x(t-T)}+\frac{\mu}{2 p_{1}}\right) \\
& -2 p_{3}\left(k_{p 3}+T k_{i 3}+\frac{\mu}{2 p_{3}}\right)\left(4 p_{1} p_{2}\left(\alpha+k_{p 1}+T k_{i 1}+\alpha k_{x(t), x(t-T)}+\frac{\mu}{2 p_{1}}\right)\left(1+k_{p 2}+T k_{i 2}+\frac{\mu}{2 p_{2}}\right)-\left(p_{1} \alpha+p_{2}\right)^{2}\right)
\end{aligned}
$$


Assuming that $\Delta_{1}<0, \Delta_{2}>0, \Delta_{3}<0$ and $p_{2}=p_{3} \beta$ for simplicity we obtain

$$
\begin{aligned}
& k_{p 1}+T k_{i 1}>\left(-\alpha-\alpha k_{x(t), x(t-T)}-\frac{\mu}{2 p_{1}}\right) \\
& k_{p 2}+T k_{i 2}>\frac{\left(p_{1} \alpha+p_{2}\right)^{2}}{4 p_{1} p_{2}\left(\alpha+k_{p 1}+T k_{i 1}+\alpha k_{x(t), x(t-T)}+\frac{\mu}{2 p_{1}}\right)}-1-\frac{\mu}{2 p_{2}} \\
& k_{p 3}+T k_{i 3}>-\frac{\mu}{2 p_{3}}
\end{aligned}
$$

Since $\alpha>0, \beta>0, a \leq k_{x(t), x(t-T)} \leq b<0$, we know that, if we choose suitable $k_{p 1}, k_{p 2}, k_{p 3}, k_{i 1}, k_{i 2}, k_{i 3}$ such that

$$
\begin{aligned}
& k_{p 1}+T k_{i 1}>\left(-\alpha(a+1)-\frac{\mu}{2 p_{1}}\right) \\
& k_{p 2}+T k_{i 2}>\frac{\left(p_{1} \alpha+p_{2}\right)^{2}}{4 p_{1} p_{2}\left(\alpha+k_{p 1}+T k_{i 1}+a \alpha+\frac{\mu}{2 p_{1}}\right)}-1-\frac{\mu}{2 p_{2}} \\
& k_{p 3}+T k_{i 3}>-\frac{\mu}{2 p_{3}}
\end{aligned}
$$

then (11) will be satisfied

By selecting $\mu=-0.2, P=\left[\begin{array}{ccc}2 & 0 & 0 \\ 0 & 14.97 & 0 \\ 0 & 0 & 1\end{array}\right], T=0.1$ we can get $k_{p 1}=2, k_{p 2}=6, k_{p 3}=0, k_{i 1}=20, k_{i 2}=40$, $k_{i 3}=0$ to satisfy (26). Fig. 2 show the effectiveness of the proposed controller which is activated from $t=20 \mathrm{~s}$.
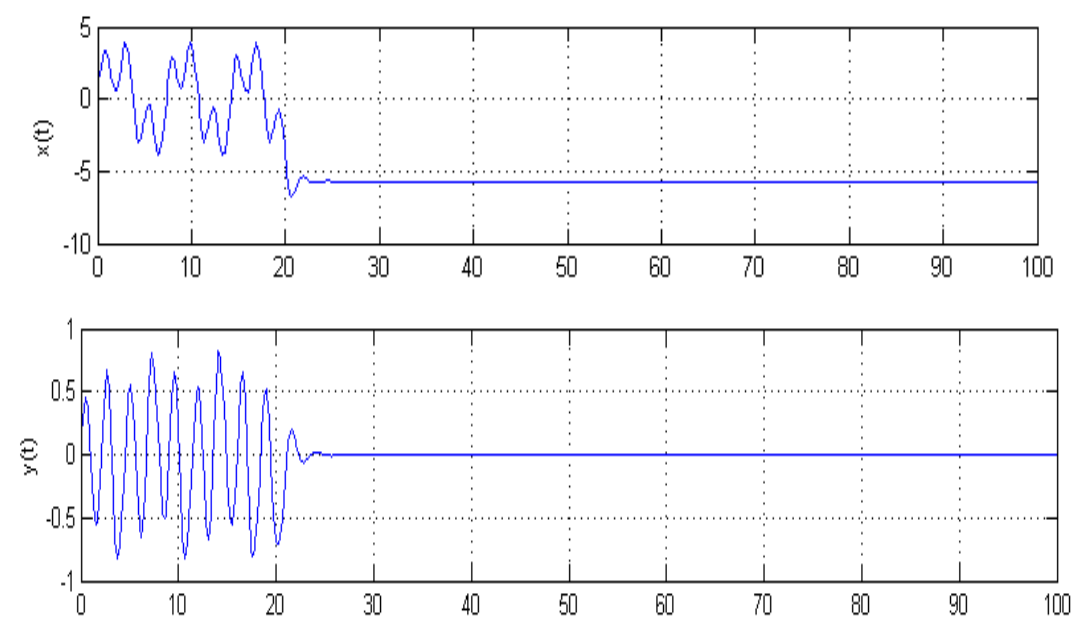


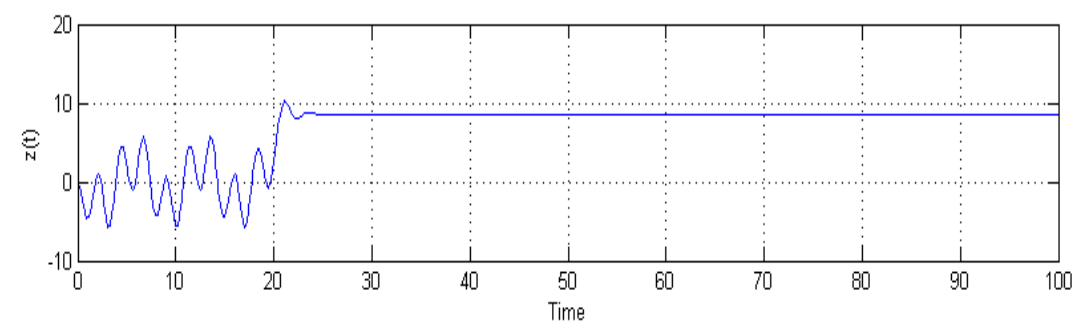

Fig. 2 the controlled states of Chua's circuit based on PI time-delayed controller with $k_{p 1}=2, k_{p 2}=6, k_{p 3}=0$

$$
\text { and } k_{i 1}=20, k_{i 2}=40, k_{i 3}=0 \text { which are activated from } t=20 \mathrm{~s}
$$

\section{CONCLUSIONS}

In this paper, proportional plus integral time-delayed feedback scheme for chaos control based on Lyapunov stabilization theory is proposed. In particular, we can find many unstable periodic orbits and stabilized them through PI time-delayed feedback control. Theoretical analysis and numerical simulation for classic Chua's circuit show the effectiveness of this technique

\section{REFERENCES}

[1] K. Pyragas, Physics Letters A 170 (1992) 421-428.

[2] M. Chen, D. Zhou, Y. Shang, Chaos Solitons Fractals 22 (2004) 1117-1125.

[3] M. Chen, Y. Shang, D. Zhou, Chaos Solitons Fractals 22 (2004) 161

[4] M. Chen, D. Zhou, Y. Shang, Physics Letters A 350 (2006) 214-220.

[5] G. Ablay, Nonlinear Analysis: Hybrid Systems 3 (2009) 531-535.

[6] C. Zhu, Nonlinear Analysis 71 (2009) 2441-2446.

[7] C. Zhu, Z. Chen, Physics Letters A 372 (2008) 4033-4036.

[8] C. Tao, C. Yang, Y. Luo, H. Xiong, F. Hu, Chaos Solitons Fractals 23 (2005) 259-263.
[9] X. Liao, P. Yu, Chaos Solitons Fractals 29 (2006) 91-107.

[10] X. Wang, L. Tian, S. Jiang, L. Yu, Journal of Information and Computing Science, 1 (2006) 2, pp 93-100 ISSN 1746-7659, England, UK.

[11] L. Fronzoni, M. Giocondo, International Journal of Bifurcation and Chaos, Vol.8, No.8 (1998) 1693-1698.

[12] Lu Jun-an, H. Baoxing, Wu Xiaoqun, Chaos Solitons Fractals 22 (2004) 229-236.

[13] X. F. Wang and G. Chen, IEEE TRANSACTIONS ON CIRCUITS AND SYSTEMS-I: FUNDAMENTAL THEORY AND APPLICATIONS, VOL.50, NO.6, JUNE 2003.

[14] Kit-Sang Tang, Kim F. Man, G. Zhong, and G. Chen, IEEE TRANSACTIONS ON CIRCUITS AND SYSTEMS-I: FUNDAMENTAL THEORY AND APPLICATIONS, VOL.48, NO.5, MAY 2001.

[15] G. JIANG and W. K. S. TANG, International Journal of Bifurcation and Chaos, Vol.12, No.10 (2002) 2239-2253

[16] G. JIANG, W. X. Zheng and G. Chen, Chaos Solitons Fractals 20 (2004) 267-275.

[17] J.M.Peña, Linear Algebra and its Applications 393 (2004) 319-332.

[18] R. FARID, A. IBRAHIM, B. ABO-ZALAM, Proceedings of the 10th WSEAS International Conference on AUTOMATION \& INFORMATION

[19] R. FARID, A. IBRAHIM, B. ABO-ZALAM, Proceedings of the 10th WSEAS International Conference on FUZZY SYSTEMS

\section{Creative Commons Attribution License 4.0 (Attribution 4.0 International, CC BY 4.0)}

This article is published under the terms of the Creative Commons Attribution License 4.0 https://creativecommons.org/licenses/by/4.0/deed.en_US 